\title{
The Application of a Novel Statistical Method for Syndromic Surveillance in England
}

\author{
Roger Morbey ${ }^{1}$, Helen Hughes ${ }^{\star 1}$, Alex Elliot ${ }^{1}$, Neville Verlander ${ }^{2}$, Nick Andrews ${ }^{2}$, Andre \\ Charlett $^{2}$ and Gillian Smith ${ }^{1}$
}

${ }^{1}$ Public Health England, Birmingham, United Kingdom; 'Public Health England, London, United Kingdom

\section{Objective}

This paper describes the design and application of a new statistical method for real-time syndromic surveillance, used by Public Health England.

\section{Introduction}

Syndromic surveillance is the real-time collection and interpretation of data to allow the early identification of public health threats and their impact, enabling public health action. ${ }^{1}$ Statistical methods are used in syndromic surveillance to identify when the activity of indicator 'signals' have significantly increased. A wide range of techniques have been applied to syndromic data internationally.

As part of the preparation for the 2012 Olympics Public Health England expanded its syndromic surveillance service. ${ }^{2}$ As new syndromic systems were introduced, statistical methods were developed and applied for each system, tailored to the particular system challenges at the time, e.g. a lack of historical data, and regular changes to geographical coverage. ${ }^{3}$

\section{Methods}

The 'Rising Activity, Multi-level Mixed effects, Indicator Emphasis' (RAMMIE) statistical method was developed in Spring 2013 and tested alongside existing methods before being applied to telehealth, ED and general practitioner (GP) syndromic surveillance systems. In August 2013 the method began to be used as the sole method for these systems and was later extended to a new GP 'in hours' system. In October 2013 the RAMMIE method was applied to a new national telehealth service available across England, NHS 111.

\section{Results}

Separate models were created for each syndromic indicator within each system because the seasonal pattern across the year and even across the week varied between indicators. A Poisson or a negative binominal model was used because the data was in the form of 'counts'; a total count was used as an offset to allow for changes in coverage and data volume. A Poisson model was used for the multi-level sub-national models as it was computationally quicker to converge, whilst the negative binominal model was preferred at the national level because it allows for potential over-dispersion.

The method has been applied successfully to syndromic systems in England providing realistic models for baseline activity and utilising prioritisation rules to ensure a manageable number of 'alarms' each day. The method is shown here to have a high sensitivity (92\%) and specificity (99\%) compared to previous methods, whilst halving the time taken to detect increased activity to 1.3 days.

\section{Conclusions}

The RAMMIE method has proved to be a reliable, effective method for generating automated alarms for syndromic surveillance. The multi-level models have enabled local models to be created for the first time across all systems and these have proved themselves to be robust across all the signals. The method is able to cope with the wide range of data volume and temporal cycles seen across the large number of signals. The prioritisation rules that reduce duplication by emphasising the most important signals help to keep alarms to manageable levels. New syndromic surveillance data sources have been incorporated into RAMMIE with a minimum of extra development work.

\section{Keywords}

Syndromic surveillance; statistical methods; risk assessment; public health action

\section{References}

1. Triple S Project. Assessment of syndromic surveillance in Europe. Lancet 2012;378:1833-4.

2. Elliot AJ, Morbey RA, Hughes HE, et al. Syndromic surveillance - a public health legacy of the London 2012 Olympic and Paralympic Games. Public Health 2013;127:777-81.

3. Morbey RA, Elliot AJ, Charlett A, et al. Development and refinement of new statistical methods for enhanced syndromic surveillance during the 2012 Olympic and Paralympic Games. Health Informatics J 2014: doi $10.1177 / 1460458213517577$.

\section{*Helen Hughes}

E-mail: helen.hughes@phe.gov.uk 